\section{Classification of sit-to-stand locomotion task based on spectral analysis of waveforms generated by accelerometric transducer}

\section{G. Costantini, M. Carota, G. Maccioni and D. Giansanti}

A new method to evaluate the ability to rise from a chair by means of the analysis of the sit-to-stand locomotion task is introduced. In particular, the method is based on the frequency analysis of the acceleration measurements supplied by a home-made transducer

Introduction: Human functional ability/disability is evaluated by carrying out some standardised simple clinical tests sometimes performed with only qualitative and/or partially quantitative observations [1]. For example, the ability to rise from a chair (sit-to-stand locomotion task) is of fundamental importance for the quality of life, being connected to the functional independence; it is commonly considered as the most mechanically demanding functional task in daily activities [2]; and in order to exactly and easily assess this motor task, quantitative measurements should then be introduced in the evaluation process. Optoelectronic or ultrasound equipments are not suitable for the application we are interested in, because of their costs and encumbrance; they require a lot of markers, which restrict the investigated movement itself and suffer shadowing effect. Instead, kinematic sensors are a valid alternative; besides resolving the above-mentioned problems, they add the necessary quantitative measurements to the qualitative observation.

In [3], it is shown that pure accelerometers (AC), also because of recent advances in miniature devices (e.g. MEMS technology), can be successfully used in human continuous monitoring such as gait analysis, sit-to-stand and stand-to-sit analysis, postural sway, fall risk.

However, little work has been reported on the use of ACs in the assessment of the sit-to-stand movement. Sit-to-stand can be automatically divided into phases and classified by identifying the sequence of two different postures; the sitting one and the standing one [4]. Fig. 1 shows the main phases of a sit-to-stand investigation, as given by a single AC affixed at the trunk at L5 level (same direction as $g$ ). It is also shown that architectures with ACs and rate gyroscopes have the advantage to better assure the feasibility of trajectory reconstruction for short locomotor tasks [5]. In this Letter we introduce a novel method for the classification of the sit-to-stand task, based on the analysis in the frequency domain of the acceleration assessed by a home-made transducer. This method can be used to discriminate between pathological and non-pathological subjects.

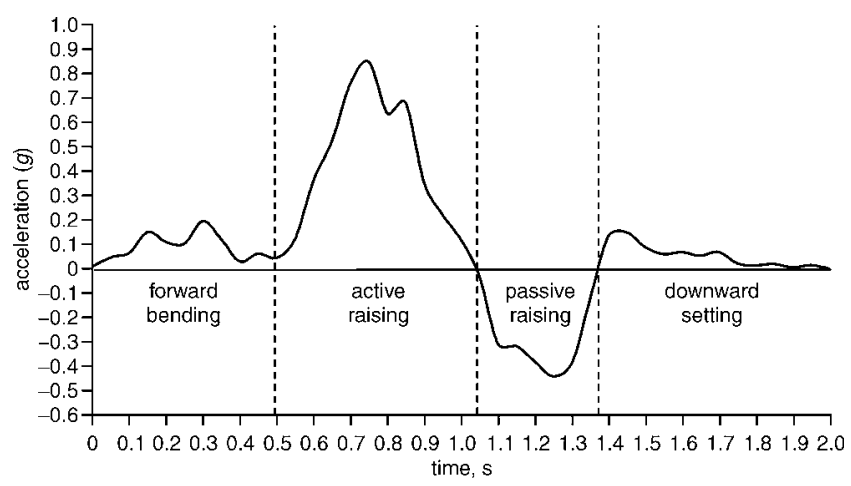

Fig. 1 Phases of the sit-to-stand

Signal processing: The mentioned transducer has been designed and built by some of the authors. It is described in detail in [5]. The core of this device (Fig. 2) is based on three accelerometric sensors (3031-Euro Sensors, US) and three rate-gyroscopic sensors, orthogonally arranged in order to derive some kinematic information. In Fig. 2, we can see on the upper-left side the sub-circuit that houses the three gyroscopes, while on the upper-right side the sub-circuit that houses the three accelerometers. The signals provided by these transducers are, respectively, the angular velocity $\omega_{S}$ and the linear acceleration $\boldsymbol{a}_{S}$. For the purpose of our specific investigation, these signals are processed according to the flow chart in Fig. 2. The filters that appear in this flow chart are second-order Sallen\&Key cells with a Butterworth behaviour and a fixed cutoff frequency of $7 \mathrm{~Hz} . C_{W}$ and $C_{A}$ are the calibration matrixes for the sensors, while $\mathbf{R}$ is the calibration matrix obtained from the components of the angular velocity [5]. Using the matrix $\mathbf{R}$ and the acceleration $\boldsymbol{a}^{\prime}$ in the mobile reference system solid to the device, we obtain the absolute acceleration $\boldsymbol{a}$ by the formula:

$$
\boldsymbol{a}=\mathbf{R} \boldsymbol{a}^{\prime}-\boldsymbol{g}
$$

where $\boldsymbol{g}$ is the gravity acceleration.

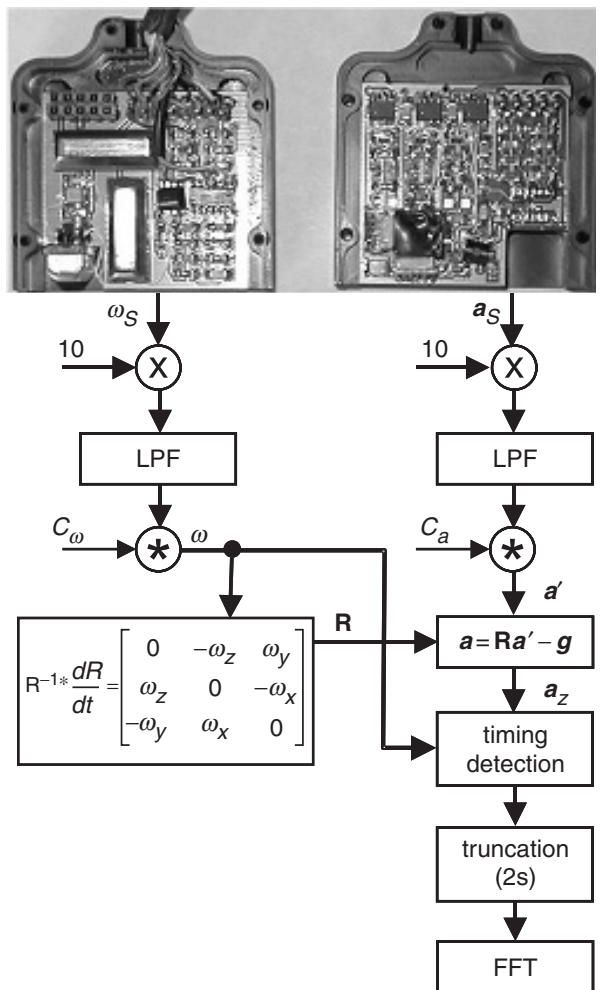

Fig. 2 Circuitry of wearable device and flow chart of signal processing

The vertical component $a_{z}$ of the acceleration (same direction as $g$ ) is taken as the waveform to be investigated in the frequency domain, by means of the following steps: 1 . detection of onset and offset times (and so its time duration) of the waveform [5];2. truncation of the waveform to fix it at $2 \mathrm{~s} ; 3$. evaluation of the spectrum of the waveform by means of the well-known FFT algorithm.

Protocol and investigation: The target of our investigation is discrimination between healthy and diseased subjects, as well as between different sit-to-stand heights, using as a starting point the measurements given by the ACs. We analysed the sit-to-stand of five healthy subjects and three Parkinsonian subjects (at first stage of pathology). The transducer was affixed at L5 level of the trunk (standard clinical position). The acceleration waveform was recorded with a sample period of $50 \mathrm{~ms}$, during $4 \mathrm{~s}$ trials, in three different conditions: A. chair height fixed to $90 \%$ of the feet-to-knee distance; B. chair height fixed to $100 \%$ of the feet-to-knee distance; C. chair height fixed to $110 \%$ of the feet-to-knee distance.

Three trials were performed for each subject and in each condition; the order of the trials was randomised. We operate on a data se obtained by analysing in the frequency domain the waveforms produced by the ACs. In particular, the features exploited in the classification phase are the frequency components of the AC generated waveforms. In Fig. 3, we report the mean values and the standard deviations, assessed over all the trials, of the DC component, the fundamental and the first three harmonics (energy is mostly distributed between these five frequency components), both for healthy and diseased subjects rising from three different chair heights. Our method is based on the fact that we can easily distinguish between different sit-to-stand typologies, regardless of the subject under test, as well as between people with different states of health (bad, good), by simply observing that every situation is labelled by a particular energy distribution over the 
frequency components. In particular, for healthy subjects, it can be noticed that:

A. rising from $90 \%$

- the DC component has a very low amplitude

- most of the energy is concentrated on the fundamental

- energy falls for the second and third harmonic

B. rising from $100 \%$

- the DC component has a generally more noticeable amplitude

- most of the energy is concentrated on the first harmonic

- little energy can be sometimes found on the third/fourth harmonic

C. rising from $110 \%$

- often, the DC component has a huge amplitude

- the maximum energy is concentrated on the first harmonic

- often, little energy can be found on the fourth harmonic

On the other hand, in case of a subject suffering from Parkinson disease, we can notice a deeply different energy distribution between frequency components:

- the DC component is always surprisingly predominant

- with reference to the three cases A, B and C, the energy distribution between the fundamental and the harmonics noticeably changes with respect to the case of healthy people.

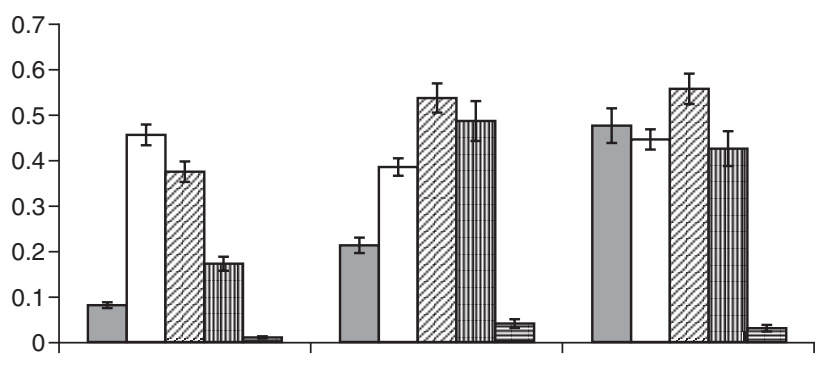

a

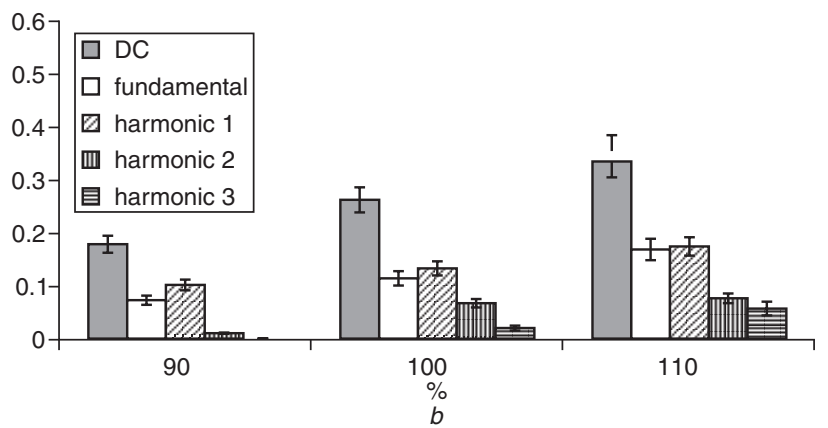

Fig. 3 Mean values and standard deviations of DC component, fundamental and first three harmonics for three different chair heights and for healthy and diseased subjects

$a$ Healthy subjects

$b$ Diseased subjects
Next step: This method will be improved by exploiting automatic classification methodologies based on adaptive systems, such as neural networks. Neural classifiers can process huge amounts of data to develop knowledge bases for the identification of particular pathologies in their early stage. Both supervised and unsupervised learning algorithms are well suited for this application and they will be investigated and applied to locomotory act classification.

(C) IEE 2006

19 July 2005

Electronics Letters online no: 20062623

doi: 10.1049/el:20062623

G. Costantini and M. Carota (Department of Electronics Engineering, University of Rome 'Tor Vergata', Via del Politecnico, 1-00133 Roma, Italy)

E-mail: costatini@uniroma2.it

G. Maccioni and D. Giansanti (Technology and Health Department, The National Health Institute, Viale Regina Elena 299, Rome, Italy)

\section{References}

1 Winters, J.M., and Crago, P.E.: 'Biomechanics and neural control of posture and movement' (Springer, New York, 2000)

2 Kralj, A.: 'Analysis of standing up and sitting down in humans: definitions and normative data presentation', J. Biomech., 1990, 23, pp. $1123-1138$

3 Mathie, J.M., et al.: 'Accelerometry: providing an integrated, practical method for long-term, ambulatory monitoring of human movement', Physiol. Meas., 2004, 25, (2), pp. R1-R20

4 Mathie, J.M., Crago, P.E., and Celler, B.G.: 'Detection of daily physical activities using a triaxial accelerometer', Med. Biol. Eng. Comp., 2003, 41,

5 Giansanti, D., Maccioni, G., and Macellari, V.: 'The development and test of a device for the reconstruction of 3D position and orientation by means of a kinematic sensor assembly with rate gyroscopes and accelerometers', IEEE Trans. Biomed. Eng., 2005, 52, (7), pp. 1271-1277 